\title{
Patterns of Hospital Admission in 60 days at Malaysian Field Hospital in Bangladesh
}

\author{
Huzairi $\mathrm{S}^{1}$, Nada S.Z2 , Alias M.Y3, Hasif $\mathrm{N}^{3}$, Shamsul B ${ }^{3}$ \\ ${ }^{1}$ Universiti Teknologi MARA, Medical Faculty, UiTM Sungai Buloh, Selangor, Malaysia; ${ }^{2}$ Pathology \\ Department, Penang General Hospital, Ministry of Health, Malaysia; ${ }^{3}$ Malaysian Field Hospital, \\ Ukhia, Bangladesh
}

\begin{abstract}
Introduction: Malaysian Field Hospital (MFH) is a tertiary healthcare centre located in Ukhia district which caters for 286 primary health clinics and 4 secondary hospitals. It provides medical services to Rohingyan refugees and the locals of Bangladesh.
\end{abstract}

Objective: To investigate admission patterns to MFH in the duration of 2 months.

Methods: This is a retrospective analysis of total admissions from March to April 2019 at MFH. Data was collected from the hospital's admissions records.

Results: There was a total of 223 admissions of which $51.1 \%$ were male and $69.5 \%$ were Rohingyans $(\mathrm{n}=155)$. Median age group was 25 years old [IQR=12-36] and median length of hospital stay was 3 days [IQR=2-4]. There was a wide distribution between disciplines with the highest number of admissions seen in the surgical department $(\mathrm{n}=86,38.6 \%)$ followed by orthopaedics $(n=58,26 \%)$, obstetrics and gynaecology $(n=51,22.9 \%)$, internal medicine $(n=26$, $11.7 \%)$ and paediatrics $(\mathrm{n}=2,0.9 \%)$.

Conclusion: Rohingyan refugees and surgical cases formed the majority of admissions. Further analysis on the specific diagnosis of these admissions is required so that preventive measures can be taken. Understanding the admission patterns to MFH is important for future healthcare strategy planning i.e. number of specialists from specific fields to deploy and therapies to supply. This is to ensure that the workforce and management rendered to patients are optimised.

Keywords: Rohingya, refugee, Malaysia Field Hospital 